\title{
Strain-encoded cardiac magnetic resonance imaging: a new approach for fast estimation of left ventricular function
}

Tomas Lapinskas ${ }^{1,2,9^{*}}$, Victoria Zieschang ${ }^{1}$, Jennifer Erley ${ }^{1}$, Lukas Stoiber ${ }^{1}$, Bernhard Schnackenburg ${ }^{3}$, Christian Stehning ${ }^{3}$, Rolf Gebker ${ }^{1,9}$, Amit R. Patel ${ }^{4}$, Keigo Kawaji ${ }^{4}$, Henning Steen ${ }^{5}$, Remigijus Zaliunas ${ }^{2}$, Sören J. Backhaus ${ }^{6,10}$, Andreas Schuster ${ }^{6,10,11}$, Marcus Makowski ${ }^{12}$, Sorin Giusca ${ }^{7}$, Grigorious Korosoglou' Burkert Pieske ${ }^{1,9}$ and Sebastian Kelle ${ }^{1,8,9^{*}}$ (D)

\begin{abstract}
Background: Recently introduced fast strain-encoded (SENC) cardiac magnetic resonance (CMR) imaging (fastSENC) provides real-time acquisition of myocardial performance in a single heartbeat. We aimed to test the ability and accuracy of real-time strain-encoded CMR imaging to estimate left ventricular volumes, ejection fraction and mass.

Methods: Thirty-five subjects (12 healthy volunteers and 23 patients with known or suspected coronary artery disease) were investigated. All study participants were imaged at 1.5 Tesla MRI scanner (Achieva, Philips) using an advanced CMR study protocol which included conventional cine and fast-SENC imaging. A newly developed realtime free-breathing SENC imaging technique based on the acquisition of two images with different frequency modulation was employed.
\end{abstract}

Results: All parameters were successfully derived from fast-SENC images with total study time of $105 \mathrm{~s}$ (a $15 \mathrm{~s}$ scan time and a $90 \mathrm{~s}$ post-processing time). There was no significant difference between fast-SENC and cine imaging in the estimation of LV volumes and EF, whereas fast-SENC underestimated LV end-diastolic mass by $7 \%$.

Conclusion: The single heartbeat fast-SENC technique can be used as a good alternative to cine imaging for the precise calculation of LV volumes and ejection fraction while the technique significantly underestimates LV enddiastolic mass.

Keywords: Cardiac magnetic resonance, Strain-encoded imaging, Cine imaging, Left ventricular function

\section{Background}

Currently, cine cardiac magnetic resonance (CMR) is the accepted standard of reference for quantification of ventricular volumes, mass and function [1]. According to recent recommendations quantification of left ventricular (LV) volumes is performed using manual contouring of the endocardial and epicardial surface from multiple short-axis planes and LV ejection fraction (EF) and mass are calculated. Although automated contouring algorithms

\footnotetext{
*Correspondence: lapinskas@dhzb.de; tomas.lapinskas@lsmuni.lt; kelle@dhzb.de

${ }^{1}$ Department of Internal Medicine / Cardiology, German Heart Center Berlin,

Augustenburger Platz 1, 13353 Berlin, Germany

Full list of author information is available at the end of the article
}

are available the majority of dedicated analysis software still relies on human interaction at least in clinical practice. In recent years, important improvements in CMR techniques have significantly reduced scan time, necessary to image the entire heart, while the duration of semiautomated volumetric analysis did not change significantly and takes up to five minutes [2].

The aim of this pilot study was to assess the ability and accuracy of recently proposed fast-SENC technique to estimate LV volumes, ejection fraction and end-diastolic mass.

C The Author(s). 2019 Open Access This article is distributed under the terms of the Creative Commons Attribution 4.0 International License (http://creativecommons.org/licenses/by/4.0/), which permits unrestricted use, distribution, and 


\section{Methods}

\section{Study population}

We prospectively invited 35 subjects (12 healthy volunteers and 23 patients with known or suspected coronary artery disease) to participate in this single center study. The coronary artery disease (CAD) was confirmed by previous quantitative coronary angiography. The study complies with the Declaration of Helsinki and was approved by the Ethics Committee of the Charité-Universitätsmedizin Berlin. All individuals were able to give written informed consent before entering the study.

\section{Cardiac magnetic resonance}

Study protocol and design details have been previously published [3]. All CMR examinations were performed on a $1.5 \mathrm{~T}$ MRI scanner (Achieva, Philips Healthcare, Best, The Netherlands) using a 5-channel phased array receiver coil in the supine position. All study participants were scanned using an identical imaging protocol.

The study protocol included an initial survey to define imaging planes. Cine images were derived using balanced steady state free precession (bSSFP) sequence with short periods of breath-holding in three LV long-axis planes. Short-axis cine images were acquired and used to calculate LV volumes, mass and ejection fraction. The following parameters were used: repetition time $(\mathrm{TR})=$ $3.3 \mathrm{~ms}$, echo time $(\mathrm{TE})=1.6 \mathrm{~ms}$, flip angle $=60^{\circ}$, acquisition voxel size $=1.8 \times 1.7 \times 8.0 \mathrm{~mm}^{3}$, and 30 phases per cardiac cycle.

A newly developed real-time free-breathing SENC imaging technique (Myocardial Solutions, Inc., Morrisville, North Carolina, USA) was employed to acquire fast-SENC images. Data were derived in three LV long-axis (two-, three- and four-chamber) views (Fig. 1a-i, respectively) and three short-axis planes at different LV levels (basal, mid-ventricular and apical). Relevant fast-SENC acquisition parameters were as follows: field-of-view $=256 \times 256 \mathrm{~mm}^{2}$, slice thickness $=10 \mathrm{~mm}$, voxel size $=4.0 \times 4.0 \times 10 \mathrm{~mm}^{3}$, single-shot spiral readout (3 interleaves) with acquisition time (TA) $=10 \mathrm{~ms}$, flip angle $=30^{\circ}$, effective echo time $(\mathrm{TE})=0.7 \mathrm{~ms}$, repetition time $(\mathrm{TR})=12 \mathrm{~ms}$, temporal resolution $=36 \mathrm{~ms}$, typical number of acquired phases $=22$, spectrally selective fat suppression (SPIR), total acquisition time per slice $<1$ s.

\section{Data analysis}

Before starting the CMR data analysis, the observers were similarly trained by a representative of the software

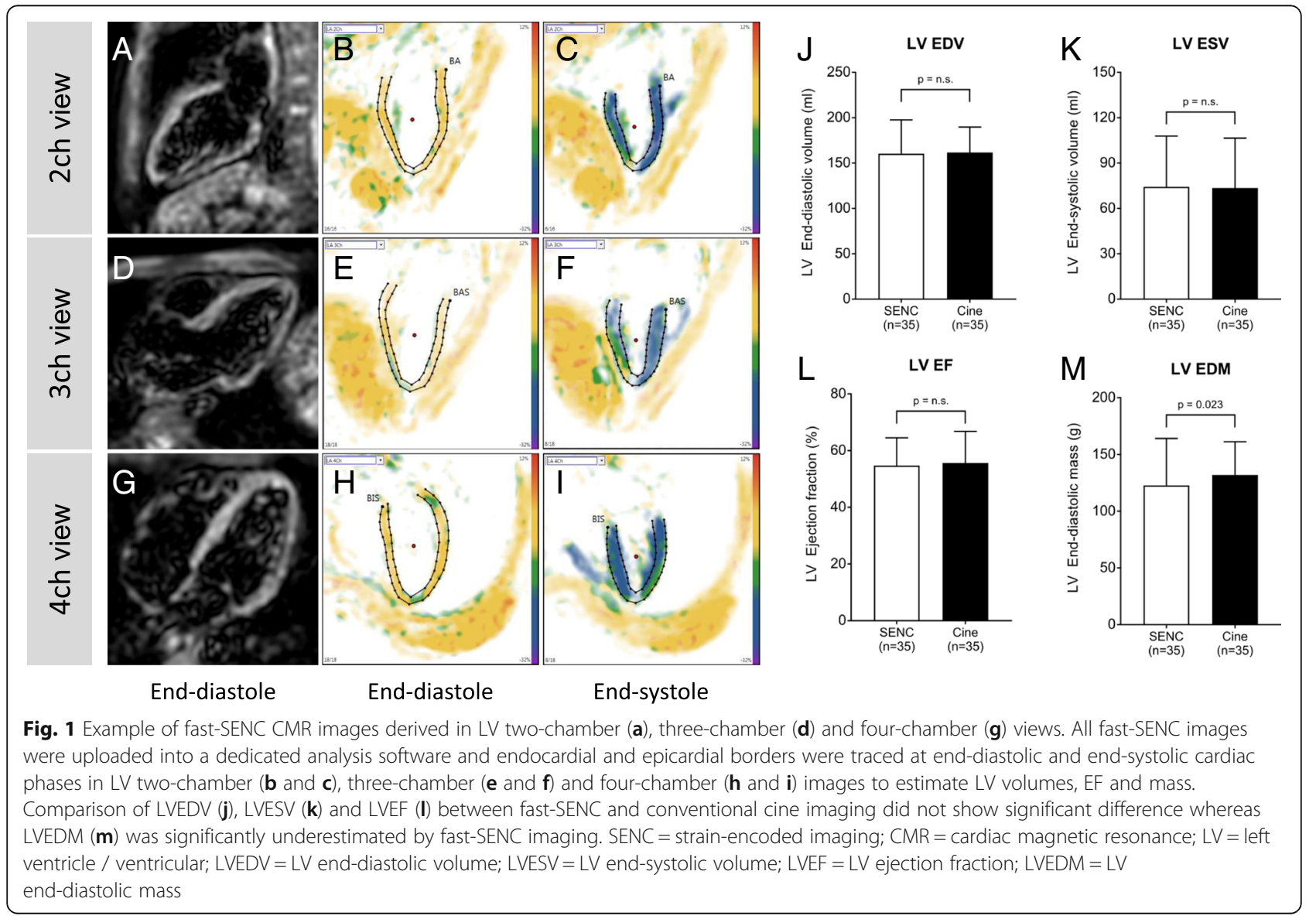


company with an emphasis on possible sources of error. All cine images were analyzed offline using Medis Suite, version 3.0 (Leiden, The Netherlands) or Virtue software (Morrisville, USA) in accordance to a recent consensus document for quantification of LV function and mass using CMR [1], while all fast-SENC images were uploaded from the scanner into a dedicated MyoStrain, version 4.2 software (Morrisville, USA). The enddiastolic and end-systolic cardiac phases were detected visually and after manual contouring of endocardial and epicardial borders LV end-diastolic (LVEDV), LV end-systolic (LVESV), LVEF and LV end-diastolic mass (LVEDM) were calculated. The LV longitudinal and circumferential strain was extracted from three LV short-axis and three LV long-axis fast-SENC images respectively. The global strain values were calculated by averaging measurements obtained from 16 segments for global longitudinal strain (GLS) and 18 segments for global circumferential strain (GCS).

\section{Statistics}

Data analysis was performed using IBM SPSS Statistics version 21.0 software (SPSS Inc., Chicago, IL, USA) for Windows. Continuous variables were expressed as mean \pm standard deviation. Differences in continuous variables were established using an unpaired Student $t$ test or Wilcoxon signed rank test depending on their distribution. Pearson's correlation coefficient was calculated to express the relation between the continuous variables. Bland-Altman analysis was performed to test if there was any bias in either CMR technique. Intraobserver and interobserver reproducibility for LVEF and LVEDM was quantified using intraclass correlation coefficient (ICC) and Bland-Altman analysis. Agreement was considered excellent for ICC $>0.74$, good for ICC $0.60-0.74$, fair for ICC $0.40-0.59$, and poor for ICC $<0.40$. Statistical significance was defined by a $p$ value $<0.05$.

\section{Results \\ Demographic data}

All study participants were able to complete the entire study protocol. Study participants with suspected or confirmed CAD were significantly older than the healthy volunteers $(61.37 \pm 10.93$ y vs. $28.67 \pm 4.89$ y, $p<0.001)$. In the CAD group there were more male subjects $(\mathrm{p}<$ 0.001 ). Healthy volunteers had significantly lower body surface area $(p=0.026)$ and body mass index $(\mathrm{p}<0.001)$. LVEDV and LVESV were similar in both groups, whereas LVSV and LVEF were significantly lower in CAD patients (LVSV: $80.48 \pm 17.81 \mathrm{ml}$ vs. $97.00 \pm 18.96$ $\mathrm{ml}, p=0.021$; LVEF: $52.09 \pm 11.29 \%$ vs. $59.75 \pm 1.36 \%$, $p=0.034)$. Subjects with CAD had significantly higher LVEDM than healthy volunteers $(143.96 \pm 30.61 \mathrm{~g}$ vs. $82.00 \pm 25.82 \mathrm{~g}, \quad p<0.001)$. Table 1 summarizes the demographic and LV functional parameters of the study population.

\section{Myocardial deformation analysis}

As described in the methods, analysis of regional myocardial deformation was performed using three long-axis fast-SENC images (for GCS) and three short-axis fast-SENC images (for GLS). LV GLS and GCS were significantly lower in CAD patients than in healthy volunteers (GLS: $-17.29 \pm 3.17 \%$ vs. $-19.34 \pm$

Table 1 Study participants' characteristics

\begin{tabular}{|c|c|c|c|}
\hline Parameter & Healthy volunteers $(n=12)$ & CAD patients $(n=23)$ & $P$ value \\
\hline \multicolumn{4}{|l|}{ Demographics } \\
\hline Age (years) & $28.67 \pm 4.89$ & $61.37 \pm 10.93$ & $<0.001$ \\
\hline Male gender & $6(50 \%)$ & $21(91 \%)$ & $<0.001$ \\
\hline $\mathrm{BSA}\left(\mathrm{m}^{2}\right)$ & $1.84 \pm 0.22$ & $1.99 \pm 0.15$ & $<0.001$ \\
\hline $\mathrm{BMI}\left(\mathrm{k} / \mathrm{m}^{2}\right)$ & $22.17 \pm 2.45$ & $26.84 \pm 2.12$ & $<0.001$ \\
\hline CAD & $0(0 \%)$ & $23(100 \%)$ & $<0.001$ \\
\hline \multicolumn{4}{|c|}{ Volumetric and functional parameters } \\
\hline LVEDV (ml) & $162.33 \pm 33.06$ & $159.17 \pm 39.98$ & 0.694 \\
\hline LVESV (ml) & $65.42 \pm 14.52$ & $78.78 \pm 39.69$ & 0.461 \\
\hline LVSV (ml) & $97.00 \pm 18.97$ & $80.48 \pm 17.81$ & 0.021 \\
\hline LVEF (\%) & $59.75 \pm 1.36$ & $52.09 \pm 11.29$ & 0.034 \\
\hline LVEDM (g) & $82.00 \pm 25.82$ & $143.96 \pm 30.61$ & $<0.001$ \\
\hline GLS (\%) & $-19.34 \pm 1.28$ & $-17.29 \pm 3.17$ & 0.034 \\
\hline GCS (\%) & $-20.21 \pm 1.48$ & $-17.67 \pm 2.63$ & 0.001 \\
\hline
\end{tabular}

Results are reported as mean \pm standard deviation or total number (percentage). CAD coronary artery disease, $B S A$ body surface area, $B M I$ body mass index, $L V$ left ventricle / ventricular; LVEDV LV end-diastolic volume, LVESV LV end-systolic volume, LVSV LV stroke volume, LVEF LV ejection fraction, LVEDM LV end-diastolic mass, GLS global longitudinal strain, GCS global circumferential strain 
$1.28, \mathrm{p}=0.034 ; \quad$ GCS: $-17.67 \pm 2.63 \pm-20.21 \pm 1.48 \%$, $p=0.001)$ (Table 1).

\section{Intermethod agreement}

SENC imaging and analysis were fast with a $15 \mathrm{~s}$ scan time and a $90 \mathrm{~s}$ post-processing time for a complete quantitative assessment. The LVEDV, LVESV and LVEF values derived from SENC images were similar as compared to those estimated using conventional cine imaging $(160.26 \pm 37.28 \mathrm{ml}$ vs. $161.54 \pm 28.17 \mathrm{ml}, p=0.928$ for LVEDV; $74.20 \pm 33.60 \mathrm{ml}$ vs. $73.43 \pm 33.04 \mathrm{ml}, p=$ 0.620 for LVESV; and $54.71 \pm 9.83 \%$ vs. $55.69 \pm 11.11 \%$, $p=0.374$ for LVEF) (Fig. 1j, k and l). However, LVEDM measured in fast-SENC images was 7\% lower when compared with estimated using cine images $(122.71 \pm 41.38 \mathrm{~g}$ vs. $131.94 \pm 29.31 \mathrm{~g}, p=0.023$ ) (Fig. $1 \mathrm{~m}$ ). Accuracy analysis demonstrated significant correlation between fast-SENC and cine imaging techniques in the measurements of LVEDV $(\mathrm{r}=0.871, p<0.001)$, LVESV $(\mathrm{r}=0.953, \mathrm{p}<0.001)$, LVEF $(\mathrm{r}=0.837, \mathrm{p}<0.001)$ and LVEDM ( $\mathrm{r}=0.864, \mathrm{p}<0.001)$ (Fig. 2a, b, c and d respectively). The Bland-Altman analysis showed narrow limits of agreement (1.96 SD) for LVEDV (Fig. 3a), LVESV (Fig. 3b), LVEF (Fig. 3c) and LVEDM (Fig. 3d).

\section{Intraobserver and interobserver reproducibility}

There was excellent intraobserver agreement for LVEF: ICC 0.976 (0.918-0.992) and LVEDM: ICC 0.983 (0.949-0.994) derived using fast-SENC technique. The analysis of interobserver reproducibility also demonstrated excellent agreement, with slightly larger limits of agreement: ICC 0.895 (0.654-0.966) for LVEF and ICC 0.846 (0.157-0.958) for LVEDM. Figure 4 demonstrates Bland-Altman analysis of intraobserver and interobserver agreement for LVEF and LVEDM.
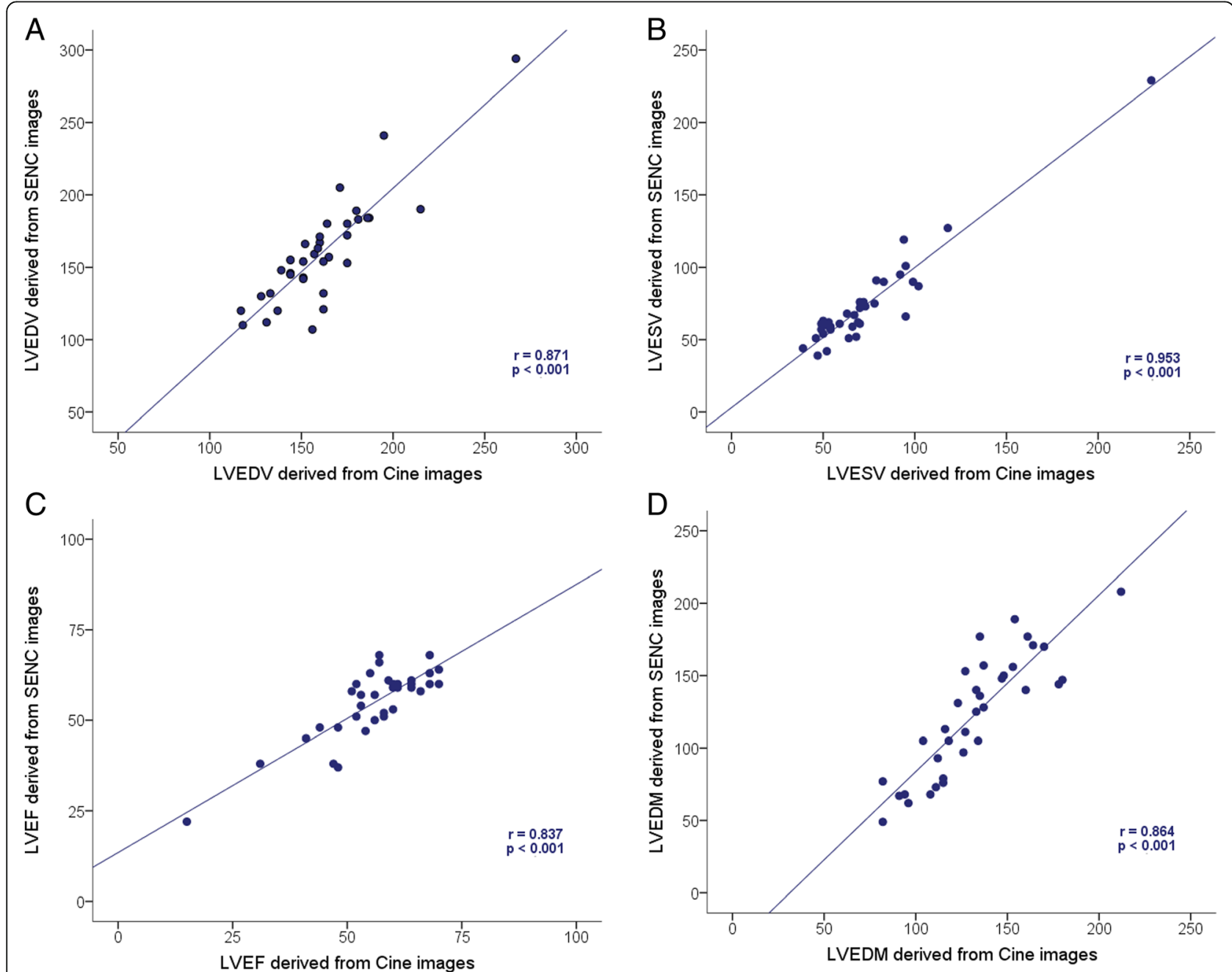

Fig. 2 Correlation analysis of LVEDV (a), LVESV (b), LVEF (c) and LVEDM (d) between fast-SENC and conventional cine imaging. LV = left ventricle / ventricular; $\mathrm{LVEDV}=\mathrm{LV}$ end-diastolic volume; $\mathrm{LVESV}=\mathrm{LV}$ end-systolic volume; $\mathrm{LVEF}=\mathrm{LV}$ ejection fraction; $\mathrm{LVEDM}=\mathrm{LV}$ end-diastolic mass; SENC = strain-encoded imaging 


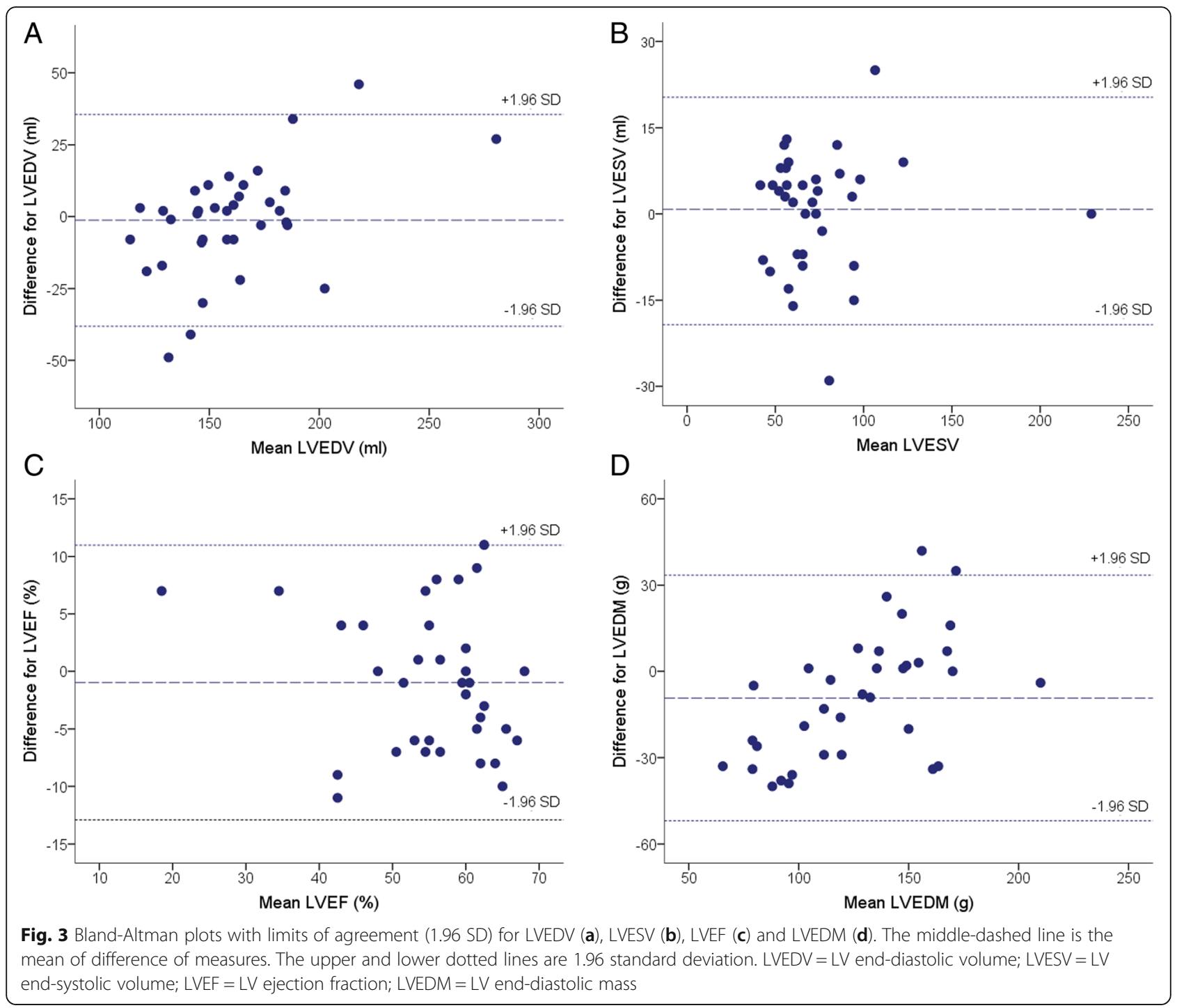

\section{Data acquisition and analysis time}

The total scan time spent to acquire CMR images using fast-SENC was $15 \mathrm{~s}$ as only six heart beats without any breath-hold are necessary to derive 3 long-axis and 3 short-axis slices. In contrast, conventional cine imaging using bSSFP sequence in a patient with heart rate of 75 beats per minute lasted approximately $45-60 \mathrm{~s}$ per slice (30 phases per cardiac cycle including breathing commands and recovery period before the next breath-hold and following slice acquisition could be started). To acquire 3 long-axis and 3 short-axis images the same process was repeated 6 times (for every slice) with a total scan duration of 270-360 s.

The time spent for data post-processing using automated contour detection algorithm or machine learning software for fast-SENC (90 s) or bSSFP (120 s) was comparable. However, total time required for data acquisition and analysis was shorter for fast-SENC technique.

\section{Discussion}

SENC is an advanced CMR technique for measuring regional myocardial function [4] as an alternative for time-consuming CMR tagging. The utility of SENC for the quantification of regional myocardial deformation has been previously validated in human $[5,6]$ and animal [7] studies. In previous studies the total scan duration for cine and SENC imaging was $30-40 \mathrm{~s}$ and for cine and CMR tagging imaging was 56-74s. Importantly, the time spent for strain analysis per patient was significantly lower using SENC (4.1 min) when compared to CMR tagging ( $9.2 \mathrm{~min}, p<0.001$ ) [8]. A further step to reduce image acquisition time was the development of the fast-SENC, which is a real-time version of SENC that shortened the scan duration to a single heartbeat [7]. These achievements provided several advantages such as elimination of breath-holds or ability to capture dynamic processes such as onset of myocardial 

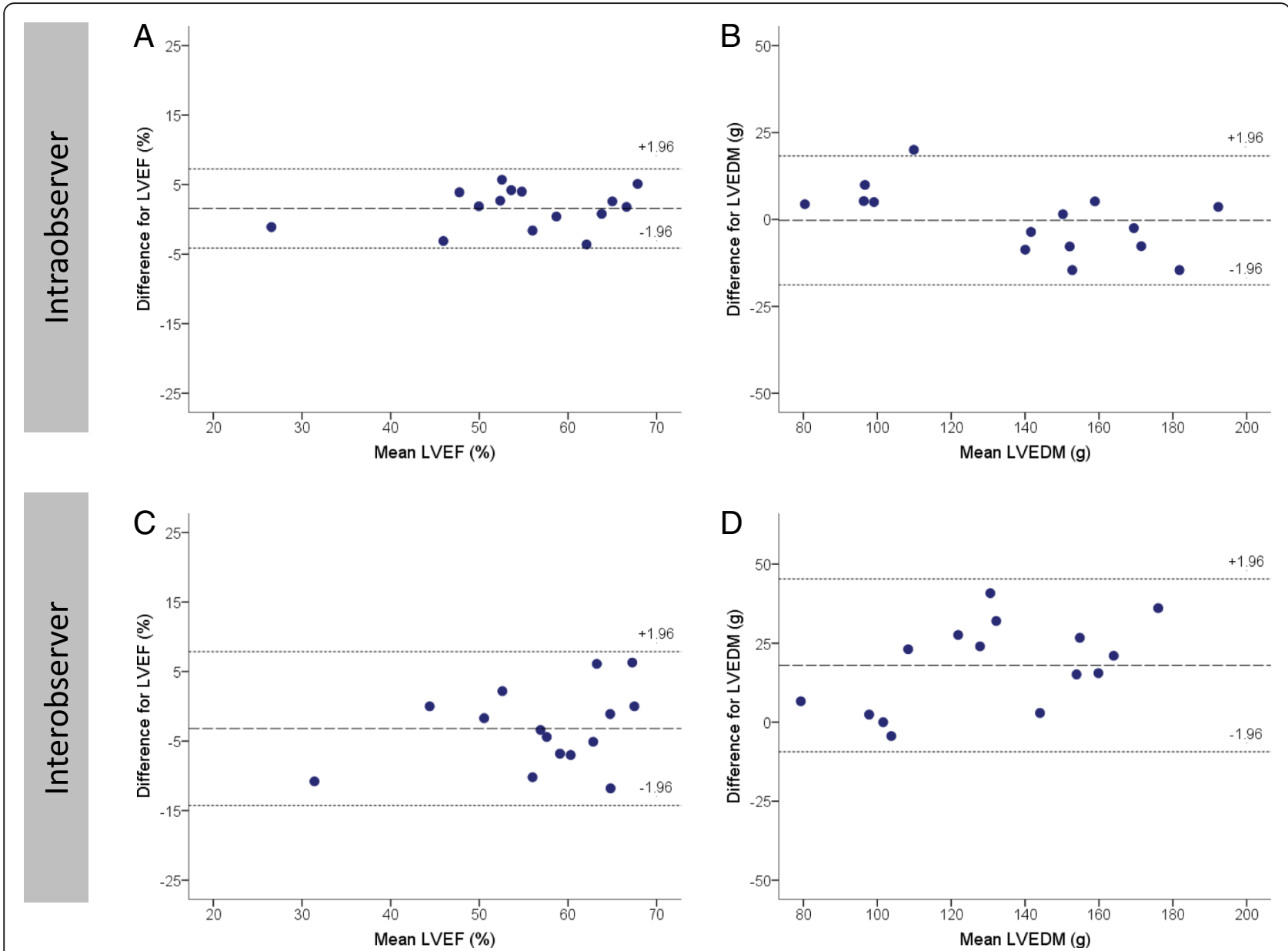

Fig. 4 Bland-Altman analysis demonstrates the intraobserver ( $\mathbf{a}$ and $\mathbf{b}$ ) and interobserver (c and $\mathbf{d}$ ) reproducibility of fast-SENC technique for LVEF ( $\mathbf{a}$ and $\mathbf{c}$ ) and LVEDM ( $\mathbf{b}$ and $\mathbf{d}$ ). The middle dashed line is the mean of difference of measures. The upper and lower dotted lines are 1.96 standard deviation. $L V E F=L V$ ejection fraction; $L V E D M=L V$ end-diastolic mass

ischemia during the stress study or cardiac arrhythmias [7]. In our study we found that using fast-SENC technique image acquisition and post-processing can be performed in less than $2 \mathrm{~min}$. There are no studies comparing the assessment of LV volumes, EF and mass using fast-SENC to other CMR imaging modalities. We did not find any relevant differences between fast-SENC and conventional cine CMR imaging regarding the estimation of LV volumes and LVEF, while fast-SENC significantly underestimated LV EDM. Recently we demonstrated that fast-SENC is a highly reproducible method for assessing LV strain [3]. In this study we found that intraobserver and interobserver agreement for LVEF and LVEDM measurements derived using fast-SENC technique is also excellent.

\section{Conclusion}

The single heartbeat fast-SENC technique can be used as a good alternative to cine imaging for the precise calculation of LV volumes and ejection fraction in the interest of time-spent, especially in patients who are unable to perform breath-holds or with cardiac arrhythmias. However, LV mass is significantly underestimated with current fast-SENC technique. LVEF and LVEDM measurements derived using fast-SENC technique are highly reproducible.

\section{Abbreviations}

CAD: Coronary artery disease; CMR: Cardiovascular magnetic resonance; EDM: End-diastolic mass; EDV: End-diastolic volume; EF: Ejection fraction; ESV: End-systolic volume; LV: Left ventricle / ventricular; SENC: Strain-encoded imaging

\section{Acknowledgements \\ We thank Anne Wölffel-Gale for editorial assistance.}

\section{Funding}

This study was not supported with specific funding that could influence study design or results. SK, AP and KK received an unrestricted research grant by Philips Healthcare and a research grant from Myocardial Solutions.

\section{Availability of data and materials}

Supporting data and materials can be obtained from the corresponding author on request. 


\section{Authors' contributions}

SK, TL, AS, AP, SG and GK conceived and designed the study. SK, TL, KK and LS carried out the experiments with help of VZ, RG, CS, and BS. BS, CS and KK were running MRI scanner to ensure acquisition of high quality CMR images. TL, VZ, JE and SG performed data processing and analysis with help of SK. LS, AS, SB and AP helped with data presentation. SG, GK, TL and VZ performed the statistical analysis. BS, HS, BP, AS, HS, SB, CS and RG provided critical revision of the manuscript. SG, GK, TL and SK wrote the manuscript with the assistance of all other co-authors. All authors read and approved the final manuscript.

\section{Ethics approval and consent to participate}

The study conforms to the ethical guidelines of the 1975 Declaration of Helsinki and was approved by the Ethics Committee of the CharitéUniversitätsmedizin Berlin. All participants gave their written informed consent to participate to the study.

\section{Consent for publication}

Not applicable.

\section{Competing interests}

All authors have read BioMed Central's guidance on competing interests. SK owns stock options of Myocardial Solutions. BS and CS are employees of Philips Healthcare. TL, SK, AS, EPK and BP received support from the DZHK (German Centre for Cardiovascular Research). All other authors declare that they have no financial and non-financial competing interest to disclose.

\section{Publisher's Note}

Springer Nature remains neutral with regard to jurisdictional claims in published maps and institutional affiliations.

\section{Author details}

'Department of Internal Medicine / Cardiology, German Heart Center Berlin, Augustenburger Platz 1, 13353 Berlin, Germany. ${ }^{2}$ Department of Cardiology, Medical Academy, Lithuanian University of Health Sciences, Kaunas, Lithuania. ${ }^{3}$ Philips Healthcare, Hamburg, Germany. ${ }^{4}$ Department of Medicine, University of Chicago, Chicago, IL, USA. ${ }^{5}$ Department of Internal Medicine / Cardiology, Marienkrankenhaus Hamburg, Hamburg, Germany. ${ }^{6}$ Department of Cardiology and Pneumology, University Medical Center, Georg-August University, Göttingen, Germany. ${ }^{7}$ Department of Cardiology and Vascular Medicine, GRN Hospital Weinheim, Weinheim, Germany. ${ }^{8}$ Department of Internal Medicine / Cardiology, Charité Campus Virchow Clinic, Berlin, Germany. ${ }^{9}$ DZHK (German Centre for Cardiovascular Research), Partner Site Berlin, Berlin, Germany. ${ }^{10}$ DZHK (German Centre for Cardiovascular Research), Partner Site Göttingen, Göttingen, Germany. ${ }^{11}$ Department of Cardiology, Royal North Shore Hospital, The Kolling Institute, Northern Clinical School, University of Sydney, Sydney, Australia. ${ }^{12}$ Department of Radiology, Charité Campus Virchow Clinic, Berlin, Germany.

\section{Received: 12 August 2018 Accepted: 21 February 2019}

Published online: 05 March 2019

\section{References}

1. Schulz-Menger J, Bluemke DA, Bremerich J, Flamm SD, Fogel MA, Friedrich MG, Kim RJ, von Knobelsdorff-Brekenhoff F, Kramer CM, Pennel DJ, Plein S, Nagel E. Standardized image interpretation and post processing in cardiovascular magnetic resonance: Society for Cardiovascular Magnetic Resonance (SCMR) board of trustees task force on standardized post processing. J Cardiovasc Magn Reson. 2013:15:35.

2. Butler SP, McKay E, Paszkowski AL, Quinn RJ, Shnier RC, Donovan JT. Reproducibility of study of left ventricular measurements with breath-hold cine MRI using a semiautomated volumetric image analysis program. Magn Reson Imaging. 1998:8(2):467-72.

3. Giusca S, Korosoglou G, Zieschang V, Stoiber L, Schnackenburg B, Stehning C, Gebker R, Pieske B, Schuster A, Backhaus S, Pieske-Kraigher E, Patel A, Kawaji K, Steen H, Lapinskas T, Kelle S. Reproducibility study on myocardial strain assessment using fast-SENC cardiac magnetic resonance imaging. Sci Rep. 2018;8(1):14100.

4. Osman NF, Sampath S, Atalar E, Prince JL. Imaging longitudinal cardiac strain on short-axis images using strain-encoded MRI. Magn Reson Med. 2001;46(2):324-34.
5. Neizel M, Lossnitzer D, Korosoglou G, Schäufele T, Lewien A, Steen H, Katus HA, Osman NF, Giannitsis E. Strain-encoded (SENC) magnetic resonance imaging to evaluate regional heterogeneity of myocardial strain in healthy volunteers: comparison with conventional tagging. J Magn Reson Imaging. 2009;29(1):99-105.

6. Korosoglou G, Lehrke S, Wochele A, Hoerig B, Lossnitzer D, Steen H, Giannitsis E, Osman NF, Katus HA. Strain-encoded CMR for the detection of inducible ischemia during intermediate stress. JACC Cardiovasc Imaging. 2010;3(4):361-71.

7. Pan L, Stuber M, Kraitchman DL, Fritzges DL, Gilson WD, Osman NF. Realtime imaging of regional myocardial function using fast-SENC. Magn Reson Med. 2006;55(2):386-95.

8. Neizel M, Lossnitzer D, Korosoglou G, Schäufele T, Peykarjou H, Steen H, Ocklenburg C, Giannitsis E, Katus HA, Osman NF. Strain-encoded MRI for evaluation of left ventricular function and transmurality in acute myocardial infarction. Circ Cardiovasc Imaging. 2009;2(2):116-22.

\section{Ready to submit your research? Choose BMC and benefit from:}

- fast, convenient online submission

- thorough peer review by experienced researchers in your field

- rapid publication on acceptance

- support for research data, including large and complex data types

- gold Open Access which fosters wider collaboration and increased citations

- maximum visibility for your research: over $100 \mathrm{M}$ website views per year

At BMC, research is always in progress.

Learn more biomedcentral.com/submissions 